\title{
Características atmosféricas do Rio de Janeiro: comparações entre as décadas de 1950 e 2000
}

\author{
Atmospheric characteristics of Rio de Janeiro: comparison between the 1950s and the \\ 2000 s
}

\author{
Natasha de Oliveira Carvalho ${ }^{1}$, Maria Gertrudes Alvarez Justi da Silva² \\ e Jéssica Lobato de Lima ${ }^{1}$ \\ ${ }^{1}$ Departamento de Meteorologia, UFRJ, Brasil \\ ${ }^{2}$ Laboratório de Meteorologia, UENF, Brasil
}

\begin{abstract}
Resumo
Os dados horários da estação meteorológica do Aeroporto do Galeão, correspondentes às décadas de 1951-1960 e 2001-2010, foram usados para comparar as variações das características da atmosfera sobre a cidade do Rio de Janeiro sobre um período de 50 anos. Foram calculadas estatísticas adequadas e utilizados testes de hipóteses adequados para garantir a significância estatística dos resultados. Distribuições probabilísticas foram também ajustadas aos dados de temperatura, umidade, pressão atmosférica e vento, sendo realçadas as diferenças encontradas na forma e na tendência central de tais distribuições. A comparação dos extremos nas temperaturas mínimas e máximas diárias entre cada década permitiu comprovar uma diminuição do número sucessivo de dias frios e aumento na frequência de máximas sucessivas acima do percentil de 90\%. Algumas hipóteses são levantadas para justificar as diferenças encontradas, sendo naturalmente a mais forte delas a crescente urbanização da cidade do Rio de Janeiro em torno do Aeroporto nos últimos 50 anos, seguida da predominância de diferenças nos padrões de variabilidade climática em cada década e do identificado aquecimento global da atmosfera obervado principalmente a partir da década de 2000.
\end{abstract}

Palavras-chave: Variabilidade climática. Clima do Rio de Janeiro. Extremos de temperatura do ar.

\begin{abstract}
The hourly data of the Galeão Airport meteorological station from the 1951-1960 and 2001-2010 decades were used to compare the variations of the atmospheric characteristics of the city of Rio de Janeiro over a period of 50 years. Suitable statistics were calculated and adequate hypothesis tests used to ensure the statistical significance of the results. The probabilistic distributions were adjusted to the temperature, humidity, atmospheric pressure and wind data and the differences in shape and central trend found in such distributions were highlighted. The comparison of the extreme minimum and maximum daily temperatures between both decades, allowed observing a decrease in the number of successive cold days and an increase in the frequency of successive maximum above the 90\% percentile. Some hypotheses were proposed to explain the differences found, the strongest obviously being the increasing urbanization of the city of Rio de Janeiro around the airport, over the last 50 years, followed by the predominance of differences in climate variability patterns in each decade and the atmosphere global warming observed mainly from the 2000s.
\end{abstract}

Keywords: Climate variability. Rio de Janeiro climate. Air temperature extremes. 


\section{Introdução}

Muito se tem escrito sobre alterações climáticas, quer relacionando-as com variações locais ou com mudanças de caráter global. Independentemente das causas, é importante a determinação da extensão destas variações ou mudanças.

A utilização da modelagem numérica da atmosfera tem sido muito útil para as caracterizações do clima e de suas variações ao nível global, mas o uso dos dados das observações meteorológicas efetuadas continuamente se mostra extremamente mais eficaz quando o que se quer é determinar as mudanças e variações de natureza local.

Com este objetivo foi feita uma análise dos dados horários coletados na estação meteorológica localizada no Aeroporto do Galeão para determinar variações no comportamento das variáveis básicas da atmosfera, eminentemente na temperatura e umidade do ar, pressão atmosférica e vento, sendo inicialmente comparados o comportamento das décadas como um todo e em seguida as variações entre as estações do ano em cada uma delas. Além disso, alguns índices recomendados para a caracterização de possíveis mudanças climáticas foram usados para análise das temperaturas mínimas e máximas diárias determinadas nesta estação meteorológica.

\section{Dados e Metodologia}

As estações meteorológicas dos aeródromos fazem observações a cada hora, incluindo medidas de pressão atmosférica, direção e velocidade do vento, temperatura e umidade do ar, visibilidade, nebulosidade, tipo, altura e base das nuvens e tempo significativo.

O Grupo de Estudos, Análises e Previsões Climáticas da UFRJ, possui em seu acervo os dados do Aeroporto do Galeão desde 1951 e, com o passar do tempo, acumulou os valores até o ano de 2010, permitindo assim que análises possam ser feitas com o detalhamento do clima em escala espaço-temporal compatível com a mesoescala.

$\mathrm{Na}$ análise pretendida neste trabalho, foram definidos dois conjuntos de dados para comparação, um agrupando as observações compreendidas entre 1951-1960 e outro contendo as observações meteorológicas entre 2001-2010.

Foram calculadas as médias e os desvios-padrões dos dados da pressão atmosférica, da temperatura do ar e do ponto de orvalho, assim como da velocidade do vento para usá-las nas estimativas dos parâmetros das distribuições probabilísticas ajustadas às variáveis meteorológicas. Para a velocidade do vento foi ajustada a distribuição Gama e para as demais variáveis foram usadas distribuições Normais ou Gaussianas. Além disso, foram formulados testes de hipóteses para verificar a significância estatística das diferenças encontradas nos parâmetros de posição, variabilidade e forma ao nível de 5\%, em todos os casos seguindo formulação de WILKS (2006).
Buscando métodos robustos e resistentes para a comparação do comportamento das características meteorológicas entre as décadas escolhidas, procedeu-se à construção de diagramas Box-Plot para as variáveis atmosféricas analisadas comparando também as estações do ano de cada década. Foram considerados os meses de janeiro, fevereiro e março como sendo verão; abril, maio e junho como outono; julho, agosto e setembro como inverno e os demais como sendo os meses de primavera.

Neste trabalho, além das comparações entre os valores médios, procedeu-se também a uma análise inicial de valores extremos para as temperaturas máximas e mínimas diárias de cada década, calculando-se alguns entre os 27 índices sugeridos pelo CLIVAR Expert Team on Climate Change Detection Monitoring and Indices (ETCCDMI) da Comissão para Climatologia da OMM (A LEXANDER et al., 2006). Especificamente, o número de dias consecutivos onde a temperatura mínima foi inferior ao percentil de $10 \%$ (CSMI), o número de dias consecutivos onde a temperatura máxima excedeu o limiar de 90\% (WSDI) e a amplitude média anual da temperatura diária (STR) foram calculados, conforme SILVA (2012) para cada década na busca da diferenciação de características entre esses períodos.

\section{Resultados}

Na Figura 1 estão representadas para cada década as distribuições Normais ajustadas aos dados de temperatura do ar, temperatura do ponto de orvalho e pressão atmosférica, assim como as distribuições Gama ajustadas aos dados da velocidade do vento, variáveis observadas a cada hora no Aeroporto do Galeão no Rio de Janeiro. Por uma inspeção inicial nas figuras são observadas variações na posição central das distribuições e no grau de espalhamento dos dados em torno deste valor central entre as décadas analisadas.

Na Tabela 1 estão apresentados os valores das médias e dos desvios-padrões das variáveis analisadas. Observa-se um aumento de $0,5^{\circ} \mathrm{C}$ na temperatura do ar e de $0,7 \mathrm{~m} / \mathrm{s}$ na velocidade do vento e diminuições de $0,2^{\circ} \mathrm{C}$ na temperatura do ponto de orvalho e cerca de $1 \mathrm{hPa}$ na pressão atmosférica. Os testes de hipóteses efetuados constataram essas diferenças como estatisticamente significativas ao nível de 5\%. Na mesma tabela revelase um aumento percentual de $13 \%$ na variabilidade da temperatura do ar e de $20 \%$ na variabilidade da temperatura do ponto de orvalho. Por outro lado a velocidade do vento teve sua variabilidade diminuída em 15\%. Mais uma vez, até mesmo pelo grande número de dados incluídos nas análises, os testes de hipóteses mostraram que essas variações observadas nos desvios padrões são estatisticamente significativas ao nível de $5 \%$. 


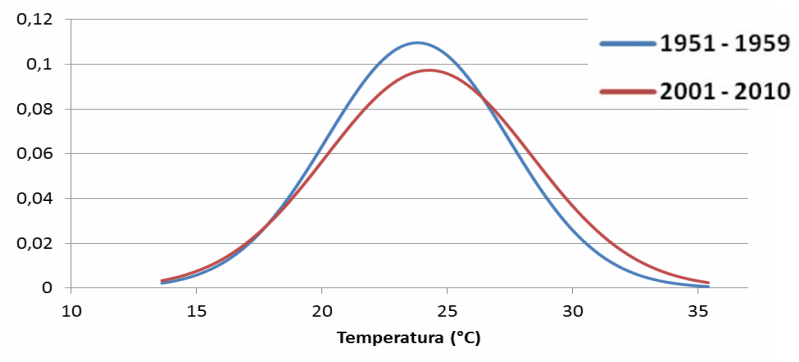

\section{(a) Temperatura do ar}

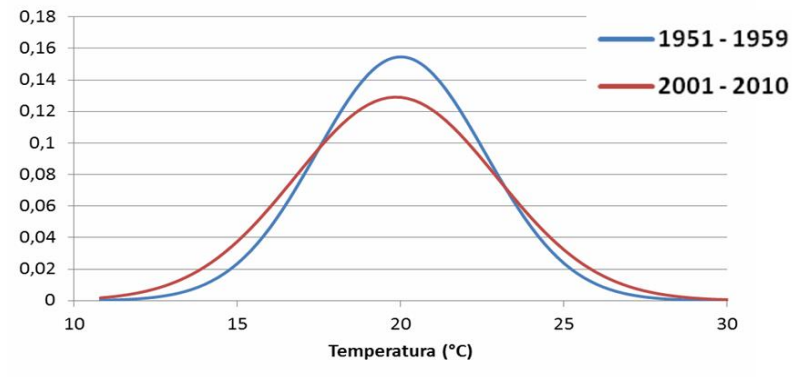

\section{(b) Temperatura do ponto de orvalho}

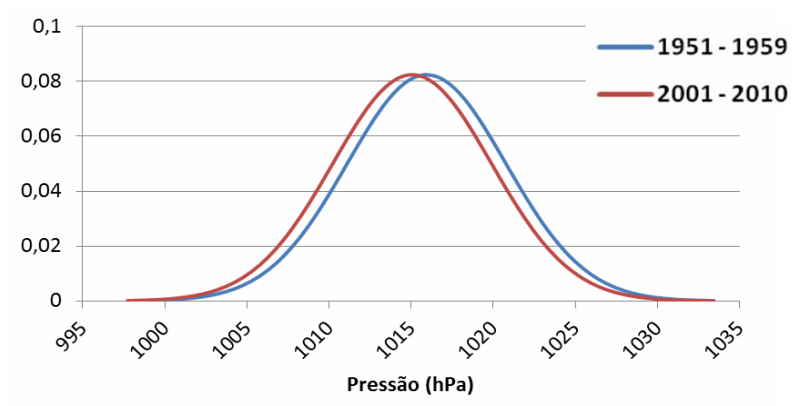

\section{(C) Pressão atmosférica}

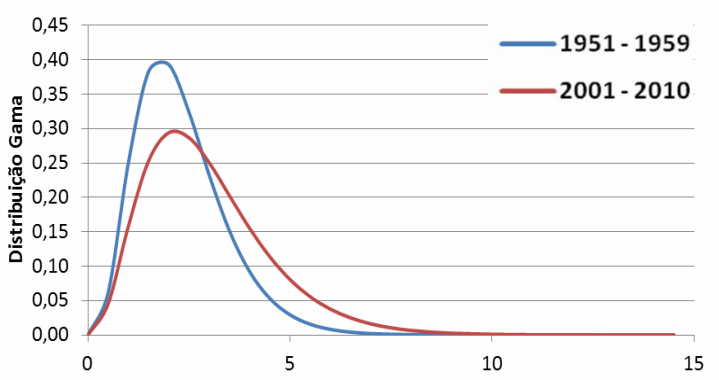

(d) Velocidade do vento

Figura 1 - Distribuições probabilísticas ajustadas aos dados horários de temperatura do ar, temperatura do ponto de orvalho, pressão atmosférica e velocidade do vento, observados no Aeroporto do Galeão no Rio de Janeiro nas décadas de 1951-1960 e 2001-2010
Tabela 1 - Estatísticas calculadas para as variáveis meteorológicas observadas no Aeroporto do Galeão

\begin{tabular}{l|c|c|c|c}
\hline \multirow{2}{*}{ Variáveis } & \multirow{2}{*}{$\mathrm{N}$} & \multicolumn{3}{|c}{ Média } \\
\cline { 3 - 5 } & & $1951-1960$ & $2001-2010$ & Diferença \\
\hline $\mathrm{T}\left({ }^{\circ} \mathrm{C}\right)$ & 82462 & 23,81 & 24,27 & 0,5 \\
\hline $\mathrm{Td}\left({ }^{\circ} \mathrm{C}\right)$ & 72686 & 20,00 & 19,85 & $-0,2$ \\
\hline $\mathrm{PNM}(\mathrm{hPa})$ & 87014 & 1015,94 & 1015,06 & $-0,9$ \\
\hline $\mathrm{WS}(\mathrm{m} / \mathrm{s})$ & 87074 & 2,29 & 2,95 & 0,7 \\
\hline \multirow{2}{*}{ Variáveis } & \multirow{2}{*}{$\mathrm{N}$} & \multicolumn{3}{|c}{ Desvio Padrão } \\
\cline { 3 - 5 } & & $1951-1960$ & $2001-2010$ & $\%$ Variação \\
\hline $\mathrm{T}\left({ }^{\circ} \mathrm{C}\right)$ & 82462 & 3,64 & 4,10 & 13 \\
\hline $\mathrm{Td}\left({ }^{\circ} \mathrm{C}\right)$ & 72686 & 2,58 & 3,09 & 20 \\
\hline $\mathrm{PNM}(\mathrm{hPa})$ & 87014 & 4,8412 & 4,8393 & 0 \\
\hline $\mathrm{WS}(\mathrm{m} / \mathrm{s})$ & 87074 & 2,21 & 1,87 & -15 \\
\hline
\end{tabular}

no Rio de Janeiro

Na Figura 2 estão representadas as rosas dos ventos destacando as distribuições das direções e velocidades observadas no Aeroporto do Galeão em cada década. As distribuições são muito semelhantes, com um leve giro mais para leste do vento predominante e uma tendência a uma maior distribuição nas frequências entre todas as direções na década de 2000 quando comparadas com a década de 1950. Não se pode descartar a hipótese de que a existência de novos obstáculos no entorno da estação meteorológica, assim como a mudança na posição da mesma ao longo dos 50 anos, possam ter causado grande parte das modificações observadas, devido à sensibilidade do vento a esses fatores.

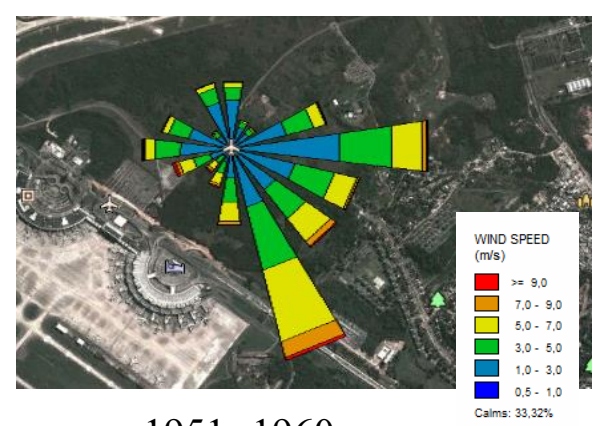

$1951-1960$

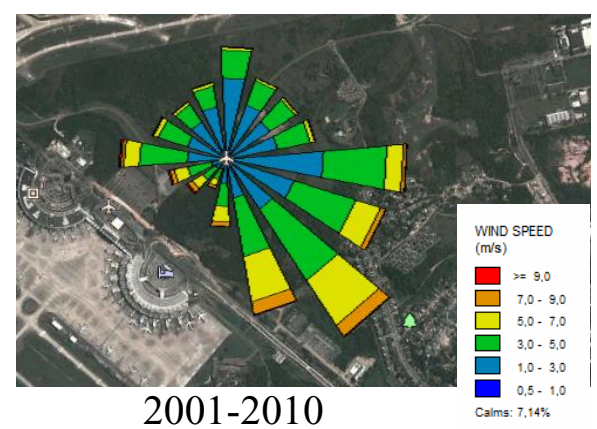

Figura 2 - Rosa dos ventos correspondentes às características observadas no Aeroporto do Galeão no Rio de Janeiro nas décadas de 1951-1960 e 2001-2010 
Na Figura 3 são comparados, através de diagramas do tipo Box-Plot, os comportamentos da temperatura do ar, do ponto de orvalho, da pressão atmosférica e da velocidade do vento nas duas décadas. Percebe-se uma diminuição nos valores da pressão atmosférica, um aumento na variabilidade da temperatura do ar e uma diminuição na variabilidade da temperatura do ponto de orvalho na década mais recente. Quanto à velocidade do vento, o diagrama evidencia um grande número de

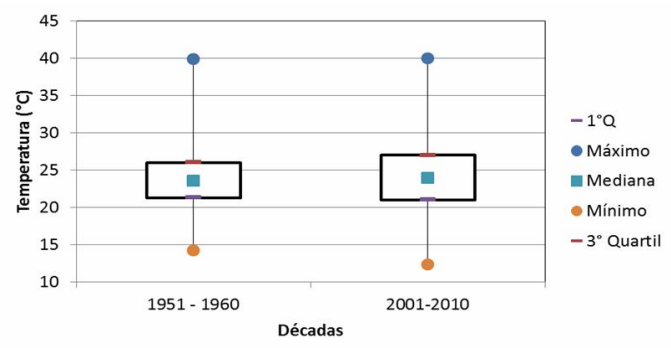

Temperatura do ar

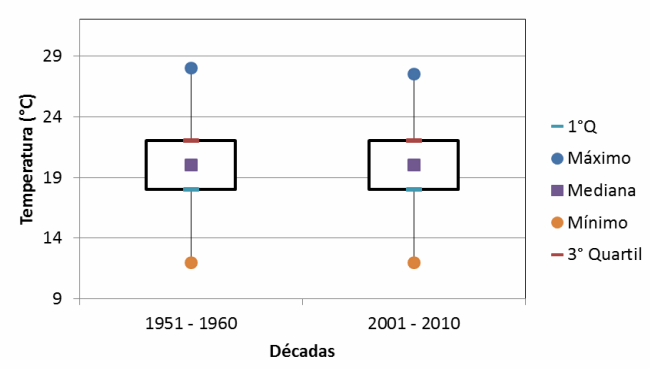

Temperatura do ponto de orvalho

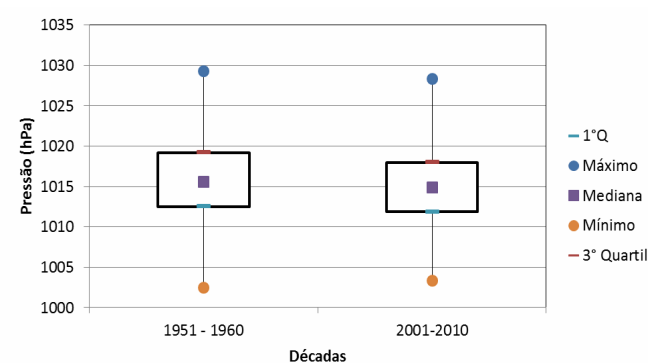

Pressão atmosférica

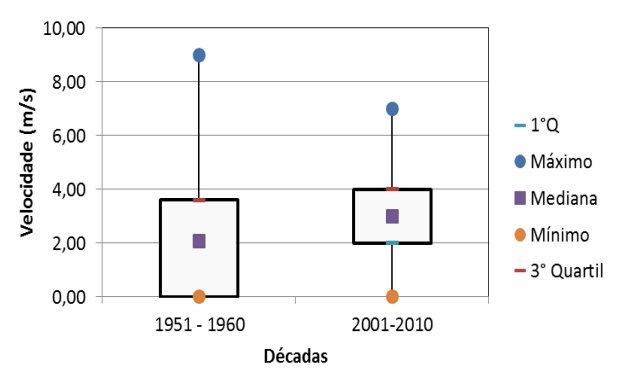

Velocidade do vento

Figura 3 - Diagramas Box-Plot para as variáveis observadas no Aeroporto do Galeão no Rio de Janeiro nas décadas de 1951-1960 e 2001-2010 observações iguais à zero na década de 1950 e valores mais extremos do que na década de 2000. Deve-se destacar que o instrumento para medir a velocidade do vento usado na primeira década era um anemômetro de placa que tem uma inércia maior que os instrumentos usados atualmente, devendo ser esta a origem das configurações obtidas.

Os diagramas apresentados na Figura 4 mostram que a pressão no verão e no inverno na década de 2001-2010 apresentou valores menores do que os da década de 1951-1960, caracterizando uma tendência a formação de baixas na região.

\section{Pressão Atmosférica - Verão}

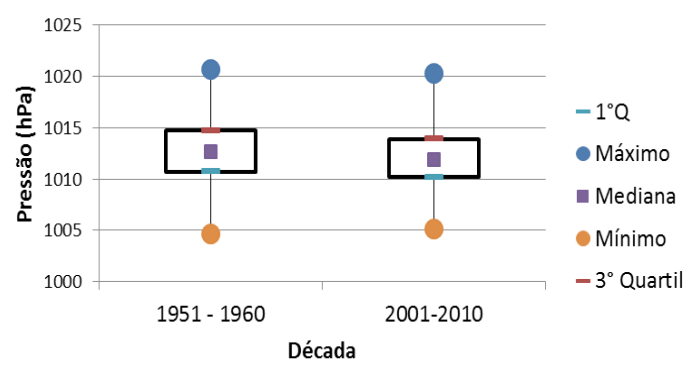

Pressão Atmosférica - Inverno

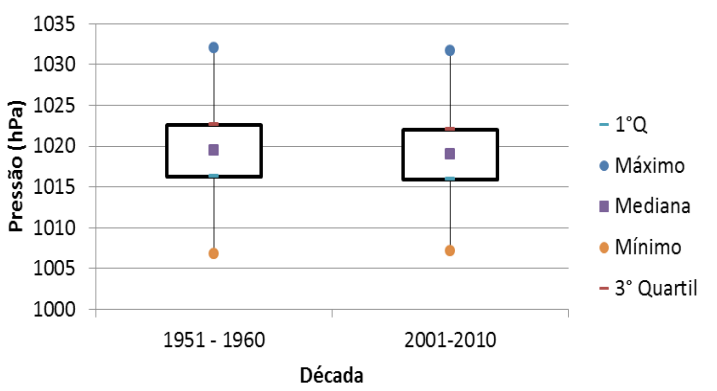

Figura 4 - Comportamento da pressão atmosférica nas décadas de 1951-1960 e 2001- 2010 no verão e no inverno no Aeroporto do Galeão do Rio de Janeiro

Por outro lado, pela Figura 5, vê-se que as temperaturas observadas no outono e no inverno se tornaram maiores e com uma maior variabilidade.

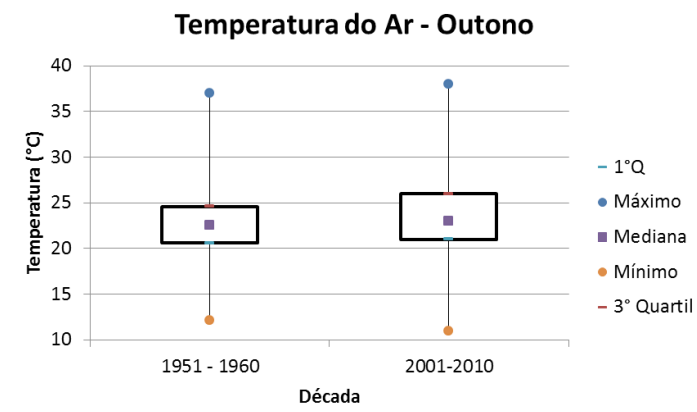

Figura 5 - Comportamento da temperatura do ar nas décadas de 1951-1960 e 2001-2010 no outono e no inverno no Aeroporto do Galeão do Rio de Janeiro

Continua... 


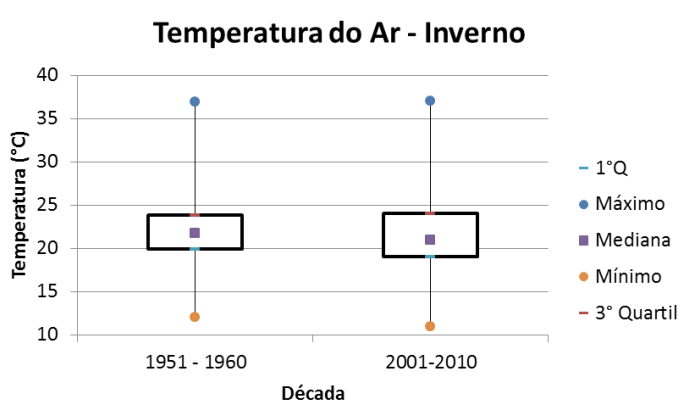

Figura 5 - Comportamento da temperatura do ar nas décadas de 1951-1960 e 2001-2010 no outono e no inverno no Aeroporto do Galeão do Rio de Janeiro Continuação

A Tabela 2 mostra os valores calculados de CSDI, WSDI e STR para as décadas de 1951-1960 e 2001-2010. Esses indicadores revelam que o número de dias frios consecutivos diminuiu e o número de dias quentes sucessivos aumentou de uma década para outra. Como já era percebido pela análise feita anteriormente em relação à temperatura do ar, o índice STR revelou que a amplitude média anual da temperatura aumentou em mais do que $1,5^{\circ} \mathrm{C}$ quando se compara a década de 1950 com a década de 2000.

Tabela 2 - Índices climáticos referentes às temperaturas extremas diárias observadas no Aeroporto do Galeão no Rio de Janeiro

\begin{tabular}{c|c|c|c}
\hline Índice & Definição & $1951-1960$ & $2001-2010$ \\
\hline CSDI & $\begin{array}{c}\text { Número máximo de dias } \\
\text { consecutivos na década com } \\
\text { TMIN < percentil 10 }\end{array}$ & 24 dias & 7 dias \\
\hline WSDI & $\begin{array}{c}\text { Número máximo de dias } \\
\text { consecutivos na década com } \\
\text { TMAX > percentil } 90\end{array}$ & 3 dias & 8 dias \\
\hline STR & $\begin{array}{c}\text { Amplitude média anual da } \\
\text { temperatura diurna }\end{array}$ & $6,8{ }^{\circ} \mathrm{C}$ & $8,4{ }^{\circ} \mathrm{C}$ \\
\hline
\end{tabular}

\section{Conclusões}

O estudo realizado permitiu avaliar as variações observadas no clima do Rio de Janeiro através da análise dos valores horários de temperatura do ar, temperatura do ponto de orvalho, pressão atmosférica e vento, observados na estação meteorológica localizada no Aeroporto do Galeão. Diferenças da ordem de $0,5^{\circ} \mathrm{C}$ na temperatura do ar, de $0,2{ }^{\circ} \mathrm{C}$ na temperatura do ponto de orvalho, de $1 \mathrm{hPa}$ na pressão e de $0,7 \mathrm{~m} / \mathrm{s}$ no vento foram observadas e revelaram-se estatisticamente significativas. Além dos valores médios, a variabilidade representada aqui pelos desvios-padrões das variáveis meteorológicas, também apresentaram um aumento proporcional da ordem de $13 \%$ e $20 \%$ para a temperatura do ar e ponto de orvalho, respectivamente, e uma diminuição de $15 \%$ na velocidade do vento, diferenças consideradas estatisticamente significativas ao nível de 5\%. O número máximo de dias consecutivos com temperaturas mínimas abaixo do percentil $10 \%$ diminuiu, assim como aumentou o número máximo de dias consecutivos com temperaturas máximas acima do percentil de $90 \%$. Além disso, a amplitude diária de temperatura aumentou na década de 2000 quando se compara com a década de 1950.

Os resultados encontrados revelam mudanças do clima local na área estudada. Muito provavelmente as diferenças são devidas às mudanças no uso do solo e da urbanização crescente no entorno da região do Aeroporto do Galeão.

Duas razões que poderiam ainda explicar parte das diferenças encontradas entre as décadas seriam (1) variações provocadas pelo aquecimento global da atmosfera nos últimos anos e (2) a superposição de padrões de variabilidade natural do clima, tais como El Niño - Oscilação Sul (ENOS), Padrão Pacífico - América do Sul (PSA), Oscilação do Atlântico Sul (SAO), Oscilação Antártica (AAO), atuando de maneiras diferentes sobre cada década e produzindo comportamentos diferentes em escala local. Em ambos os casos, estudos mais específicos seriam necessários para a apuração mais precisa da parte da responsabilidade de cada um destes efeitos sobre as variações observadas.

\section{Agradecimentos}

As autoras agradecem aos meteorologistas Vinícius Albuquerque de Almeida, pela ajuda na aquisição dos dados, e Wanderson Luiz Silva, pela ajuda na determinação dos índices climáticos.

\section{Referências}

ALEXANDER, L. V.X.ZHANG, T.C.PETERSON, J. CAESAR, B. GLEASON, A. KLEIN TANK, M. HAYLOCK, D. COLLINS, B. TREWIN, F. RAHIMZADEH, A. TAGIPOUR, P.AMBENJE, K. RUPA KUMAR, J.REVADEKAR, G. GRIFFITHS, L. VINCENT, D. STEPHENSON, J. BURN, E. AGUILAR, M. BRUNET, M. TAYLOR, M. NEW, P. ZHAI, M. RUSTICUCCI, J. L. VAZQUEZAGUIRRE. Global observed changes in daily climate extremes of temperature and precipitation. Journal of Geophysical Research-Atmospheres, v. 111, p. 19842012, 2006.

SILVA, W. L. Tendências observadas e projeções futuras de extremos climáticos na cidade do Rio de Janeiro. Monografia de Conclusão de Curso, Universidade Federal do Rio de Janeiro, 85 p, 2010.

WILKS, D. S. Statistical methods in the Atmospheric Sciences. 2. Ed. San Diego, Academic Press, 627 p, 2006. 